\title{
Development of An inventory model with Volume flexibility, random deterioration and increasing exponentially demand rate
}

\author{
Dr. Ravish Kumar Yadav ${ }^{1}$, Ms. Pratibha Devi² \\ 1. Associate Professor, Department of Mathematics, Hindu College, Moradabad \\ ${ }^{2,}$ Research Scholar, Department of Mathematics, Hindu College ,Moradabad
}

\begin{abstract}
A volume flexible inventory model is developed. This model is developed for deteriorating item by assuming that the deterioration rate is depend on a parameter and time. The production rate is variable, production cost become a function of production rate, unit cost depending upon material cost, labour cost and tool cost. The demand rate is increasing exponentially shortages are allowed. Cost minimization technique used to find out optimal values for different inventory variables.
\end{abstract}

Key words: volume flexibility, deterioration

\section{Introduction:}

This study presents a production inventory model with a time dependent random deterioration function and increasing exponentially demand over a fix time horizon with the finite. The shortages allow and excess demand is backlogged. Expressions for optimal parameter are obtained .We also obtained Production scheduling period, maximum inventory level and total average cost.

An inventory system the effect of deterioration plays an important role. Deterioration is derived as decay or damage such that the item cannot be used for its original propose. Foods, pharmaceuticals, chemicals, blood, drugs are a few examples of such items in which sufficient deterioration can take place during the storage period of the units and the importance of this loss must be taken into account when analyzing the system.

In this paper we present a realistic inventory model in which the production rate is variable and demand is an exponentially increasing function time and deterioration is random function says that deterioration of an item depends upon the fluctuation of humidity, temperature, etc. It would be more reasonable and realistic if we assume the deterioration function $\theta$ to depend upon a parameter " $\alpha$ " in addition to time $t$.

When describing optimum policies for deteriorating items Ghare and Schrader (1963) proposed a constant rate of deterioration and constant rate demand. In recent year, inventory problem for deterioration items have been widely studied after Ghare and Schrader (1963), Covert and Philip (1973) formulated the model for variable deterioration rate with two parameters Weibull disturbation Goswami and Chaudhuri (1991), Bose et al (1995) assumed either instantaneous or finite production with different assumption on the pattern of deterioration.

Balkhi and Benkheroot (1996) considered a production a production lot size inventory model with arbitrary production and demand rate depends on the time function.

Bhunia and Maiti's (1977) model to formulate a production inventory model. Chang and Deve (1999) investigated an EOQ model allow shortage and backlogging. It is assumed that the backlogging rate is variable and dependent on the length of waiting time for the next replenishment. Recently, many researchers have modified inventory policies by considering the "time proportional partial backlogging rate" such as Wang (2002), Perumal (2002), Teng et al (2003), Skouri and Papachristos (2003) and Kun-Shan et al (2005) etc.

In the Classical Economic Production Lot Size(EPLS) model, the production rate of a machine is regarded to be pre-determinded and inflexible1.Alder and Nanda, Sule, Axsater and Elmaghraby, Muth and Spearmann extended the EPLS model to situations where learning effects would induce an increase in the production rate. Proteus7, Rosenblat and Lee and Cheng considered the EPLS model in an imperfect production process in which the demand would exceed the supply. Schweitzer and Seidmann adopted, for the first time, the concept of flexibility in the machine production rate and discussed optimization of processing rates for a FMS (flexible manufacturing system). Obviously, the machine production rate is a decision variable in the case of a FMS and then the unit production cost becomes a function of the production rate. Khouja and Mehrez and Khouja extended the EPLS model to an imperfect production process with a flexible production rate. Silver, Moon, Gallego and Simchi Levi discussed the effects of slowing down production in the context of a manufacturing equipment of a family of items, assuming a common cycle for all the items. Gallego extended this model by removing the stipulation of a common cycle for all the items. But the above studies did not consider the demand rate to be variable. It is a common belief that large piles of goods displayed in a supermarket lead the customers to buy more. Volume flexibility is a major component in a FMS. The 
manufacturing flexibility which is capable of adjusting the production rate with the variability in the market demand is known as volume flexibility.

S. Sana and K. S. Chaudhuri, consider a volume flexible manufacturing system for a deteriorating item with an inventory-level-dependent demand rate. They find tha the demand rate remains stock-dependent for some time and then becomes a constant after the stock falls down to a certain level. Several factors like limited number of potential customers and their goodwill, price and quality of the goods, locality of shop, etc. can be accounted for the change in the demand pattern

This model is developed for deteriorating item by assuming that the deterioration rate is depend on a parameter and the production rate is variable. The demand rate is increasing exponentially. Cost minimization technique used to find out optimal values for different inventory variables.

\section{Assumption and Notation:}

The mathematical model of the production inventory problem considered herein is developed on the basic of the following assumptions-:

(a) A single item is considered over a prescribed period of $\mathrm{T}$ units of time, which is subject to a time dependent Random deterioration rate.

(b) Demand rate $\mathrm{D}(\mathrm{t})$ is known and increasing exponentially $\mathrm{D}(\mathrm{t})=A \mathrm{e}^{\lambda \mathrm{t}}, \mathrm{t} \geq 0$, $\mathrm{A}$ is initial demand, $\lambda$ is a constant governing the increasing rate of demand.

(c) Production rate $\mathrm{K}$ is variable.

(d) Deterioration of the units is considered only after they have been received into the inventory.

(e) No replacement or repair of deteriorated item is made during given cycle.

(f) Shortages are allowed and backlogged.

\section{Notation :}

(1) $\quad \mathrm{I}(\mathrm{t})=$ Inventory level at any time $\mathrm{t}, \mathrm{t} \geq 0$

(2) $\theta=\theta(\alpha) t$, the items deterioration rate is random.

(3) $\quad \mathrm{I}_{\mathrm{m}}=$ Maximum inventory level.

(4) $\mathrm{I}_{\mathrm{b}}=$ Unfilled order backlog.

$\eta(K)=R+\frac{G}{K}+H K=$ Unit production cost, $\left[\eta(K)=R+\frac{G}{K}+H K\right]$ is depends upon the production rate, where $\mathrm{R}, \mathrm{G}$ and $\mathrm{H}$ are the material cost, labour cost and tool or dye cost respectively.

(6) $\mathrm{C}=$ Set up cost

(7) $\quad C_{D}=$ The cost of a deteriorated item.

(8) $C_{h}=$ Inventory carrying cost per unit per month.

(9) $\quad \mathrm{C}_{\mathrm{S}}=$ Shortage cost per unit.

(10) $\mathrm{T}=\left(t_{1}+t_{2}+t_{3}+t_{4}\right)$ is the cycle time.

(11) $\quad \mathrm{C}_{\mathrm{T}}=$ The total average cost of system.

\section{Mathematical Model :}

Initially, the inventory level is start with zero. The production inventory level starts at time $\mathrm{t}=0$ and it reaches at maximum inventory level $\mathrm{I}_{\mathrm{m}}$ after $\mathrm{t}_{1}$ time unit. At that time production is stopped and the inventory level is decreasing continuously and reaches zero at time $t_{2}$ at this time shortages start developing at time $t_{3}$ it reaches to maximum shortage level $\mathrm{I}_{\mathrm{b}}$. This time fresh production start to remove backlog by the time $t_{4}$. Here our aim is to find out the optimal values of $t_{1}, t_{2}, t_{3}, t_{4}, I_{m} \& I_{b}$ that minimize the total average cost $\left(C_{T}\right)$ over the time horizon $(0, \mathrm{~T})$.

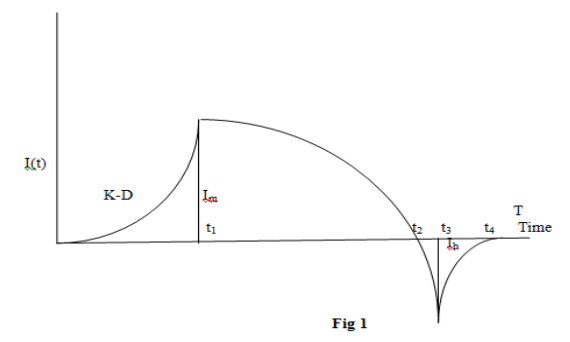

The differential equation governing the stock status during the period $0 \leq \mathrm{t} \leq \mathrm{T}$ can be written as 


$$
\begin{array}{ll}
\frac{d I(t)}{d t}+\theta(\alpha) t I(t)=K-A e^{\lambda t}, & 0 \leq t \leq t_{1} \\
\frac{d I(t)}{d t}+\theta(\alpha) t I(t)=-A e^{\lambda t}, & 0 \leq t \leq t_{2} \\
\frac{d I(t)}{d t}=-A e^{\lambda t}, \quad 0 \leq t \leq t_{3} & \\
\frac{d I(t)}{d t}=K-A e^{\lambda t}, \quad 0 \leq t \leq t_{4} &
\end{array}
$$

Using the boundary condition

$\mathrm{I}(\mathrm{t})=0$ at $\mathrm{t}=0$, T and $\mathrm{t}_{1}+\mathrm{t}_{2}$

(5)

$\mathrm{I}\left(\mathrm{t}_{2}\right)=\mathrm{I}_{\mathrm{m}}$ and $-\mathrm{I}\left(\mathrm{t}_{3}\right)=\mathrm{I}_{\mathrm{b}}$

Solution of equation (1), (2), (3) and (4) by adjusting the constant of integration using boundary condition are given by

$$
\begin{aligned}
I(t)= & e^{-\theta(\alpha) t^{2} / 2}\left[K\left\{t+\frac{\theta(\alpha) t^{3}}{6}\right\}-\frac{A e^{\lambda t}}{\lambda}\left\{1+\frac{\theta(\alpha) t^{2}}{2}-\frac{\theta(\alpha) t}{\lambda}+\frac{\theta(\alpha)}{\lambda^{2}}\right\}+\frac{A}{\lambda}\left\{1+\frac{\theta(\alpha)}{\lambda^{2}}\right\}\right] \\
I(t)= & A\left[\frac{e^{\lambda t_{2}}}{\lambda} e^{-\theta(\alpha) t_{2}{ }^{2} / 2}\left\{1+\frac{\theta(\alpha) t_{2}^{2}}{2}-\frac{\theta(\alpha) t_{2}}{\lambda}+\frac{\theta(\alpha)}{\lambda^{2}}\right\}\right. \\
& \left.-\frac{e^{\lambda t}}{\lambda} e^{-\theta(\alpha) t^{2} / 2}\left\{1+\frac{\theta(\alpha) t^{2}}{2}-\frac{\theta(\alpha) t}{\lambda}+\frac{\theta(\alpha)}{\lambda^{2}}\right\}\right], 0 \leq t \leq t_{2} \\
I(t)= & \frac{A}{\lambda}\left(1-e^{\lambda t}\right), 0 \leq t \leq t_{3}
\end{aligned}
$$

(9)

$$
I(t)=K\left(t-t_{4}\right)-\frac{A}{\lambda}\left(e^{\lambda t}-e^{\lambda t_{4}}\right), 0 \leq t \leq t_{4}
$$

The inventory level of production start initially at time unit $t=0$ to $t=t_{1}$ at maximum level $I_{m}$ is obtained using equation (5), (7) and (8).

$$
\begin{aligned}
& I_{m}=e^{-\theta(\alpha) \mathrm{t}_{1}{ }^{2} / 2}\left[K\left\{\mathrm{t}_{1}+\frac{\theta(\alpha) \mathrm{t}_{1}^{3}}{6}\right\}-\frac{A e^{\lambda \mathrm{t}_{1}}}{\lambda}\left\{1+\frac{\theta(\alpha) \mathrm{t}_{1}{ }^{2}}{2}-\frac{\theta(\alpha) \mathrm{t}_{1}}{\lambda}+\frac{\theta(\alpha)}{\lambda^{2}}\right\}+\frac{A}{\lambda}\left\{1+\frac{\theta(\alpha)}{\lambda^{2}}\right\}\right] \\
& =A\left[\frac{e^{\lambda t_{2}}}{\lambda} e^{-\theta(\alpha) t_{2}{ }^{2} / 2}\left\{1+\frac{\theta(\alpha) t_{2}{ }^{2}}{2}-\frac{\theta(\alpha) t_{2}}{\lambda}+\frac{\theta(\alpha)}{\lambda^{2}}\right\}-\frac{1}{\lambda}\left\{1+\frac{\theta(\alpha)}{\lambda^{2}}\right\}\right]
\end{aligned}
$$

and after time $t_{1}$ the production is stopped and stock level is decreasing continuously and become zero at time $\mathrm{t}=\mathrm{t}_{2}$ at that time shortages are develop and reaching to $\mathrm{I}_{\mathrm{b}}$ at time $\mathrm{t}=\mathrm{t}_{3}$ is obtained by equation (6)

$$
I_{b}=\frac{A}{\lambda}\left(1-e^{\lambda t_{3}}\right)=-K t_{4}-\frac{A}{\lambda}\left(1-e^{\lambda t_{4}}\right)
$$

Thus by equation (11) we observed that $t_{1}$ and $t_{2}$ are dependent so they are related by the equation

$$
t_{2}=f\left(t_{1}\right)
$$

and by equation (12) $t_{3}$ and $t_{4}$ are dependent to each other so related by the equation

$$
t_{3}=g\left(t_{4}\right)
$$


Total amount of deteriorated units $\left(\mathrm{I}_{\mathrm{D}}\right)$ during the period $(0, \mathrm{~T})$ is given by

$$
\begin{aligned}
& I_{D}=\int_{0}^{t_{1}} \theta(\alpha) t I(t) d t+\int_{0}^{t_{2}} \theta(\alpha) t I(t) d t \\
& =\int_{0}^{t_{1}} \theta(\alpha) t e^{-\theta(\alpha) t^{2} / 2}\left[\begin{array}{l}
K\left\{t+\frac{\theta(\alpha) t^{3}}{6}\right\}-\frac{A e^{\lambda t}}{\lambda}\left\{1+\frac{\theta(\alpha) t^{2}}{2}-\frac{\theta(\alpha) t}{\lambda}+\frac{\theta(\alpha)}{\lambda^{2}}\right\} \\
+\frac{1}{\lambda}\left\{1+\frac{\theta(\alpha)}{\lambda^{2}}\right\}
\end{array}\right] d t+ \\
& \int_{0}^{t_{2}} \theta(\alpha) t e^{-\theta(\alpha) t^{2} / 2}\left[\frac{A e^{\lambda t_{2}}}{\lambda}\left\{1+\frac{\theta(\alpha) t_{2}^{2}}{2}-\frac{\theta(\alpha) t_{2}}{\lambda}+\frac{\theta(\alpha)}{\lambda^{2}}\right\}\right. \\
& \left.-\frac{A e^{\lambda t}}{\lambda}\left\{1+\frac{\theta(\alpha) t^{2}}{2}-\frac{\theta(\alpha) t}{\lambda}+\frac{\theta(\alpha)}{\lambda^{2}}\right\}\right] d t \\
& =\left[\left\{K \theta(\alpha)\left(\frac{t_{1}^{3}}{6}-\frac{\theta(\alpha) t_{1}^{5}}{15}-\frac{\theta^{2}(\alpha) t_{1}^{7}}{84}\right)\right\}\right]+\frac{A \theta(\alpha)}{\lambda}\left(1+\frac{\theta(\alpha)}{\lambda^{2}}\right)\left(\frac{t_{1}^{2}}{2}-\frac{\theta t_{1}^{4}}{8}\right) \\
& -\frac{A \theta(\alpha) e^{\lambda t_{1}}}{\lambda}\left(1+\frac{\theta(\alpha)}{\lambda^{2}}\right)\left[\frac{t_{1}}{\lambda}-\frac{1}{\lambda^{2}}-\frac{\theta(\alpha)}{2}\left(\frac{t_{1}^{3}}{\lambda}-\frac{3 t_{1}^{2}}{\lambda^{2}}+\frac{6 t_{1}}{\lambda^{3}}-\frac{6}{\lambda^{4}}\right)\right] \\
& {\left[t_{1}\left(\frac{t_{1}^{3}}{\lambda}-\frac{t_{1}^{2}}{\lambda^{2}}+\frac{6 t_{1}}{\lambda^{3}}-\frac{6}{\lambda^{4}}\right)-\frac{\theta(\alpha)}{2}\right.} \\
& -\frac{A \theta^{2}(\alpha) e^{\lambda t_{1}}}{2 \lambda}\left(\frac{t_{1}^{5}}{\lambda}-\frac{5 t_{1}^{4}}{\lambda^{2}}-+\frac{20 t_{1}^{3}}{\lambda^{3}}-\frac{60 t_{1}^{2}}{\lambda^{4}}+\frac{120 t_{1}}{\lambda^{5}}-\frac{120}{\lambda^{6}}\right) \\
& {\left[-\frac{2}{\lambda}\left(\frac{t_{1}^{2}}{\lambda}-\frac{2 t_{1}}{\lambda^{2}}+\frac{2}{\lambda^{3}}\right)+\frac{\theta(\alpha)}{\lambda}\left(\frac{t_{1}^{4}}{\lambda}-\frac{4 t_{1}^{3}}{\lambda^{2}}+\frac{12 t_{1}^{3}}{\lambda^{3}}-\frac{24 t_{t_{1}}}{\lambda^{4}}+\frac{24}{\lambda^{5}}\right)\right]} \\
& +\left[\frac{\theta(\alpha) A e^{\lambda t_{2}}}{\lambda} e^{-\theta(\alpha) t_{2}{ }^{2} / 2}\left(1+\frac{\theta(\alpha) t_{2}^{2}}{2}-\frac{\theta(\alpha) t_{2}}{\lambda}-\frac{\theta(\alpha)}{\lambda}\right) \frac{t_{2}^{2}}{2}\right] \\
& -\frac{A \theta(\alpha) e^{\lambda t_{2}}}{\lambda}\left(1+\frac{\theta(\alpha)}{\lambda^{2}}\right)\left[\frac{t_{2}}{\lambda}-\frac{1}{\lambda^{2}}-\frac{\theta(\alpha)}{2}\left(\frac{\mathrm{t}_{2}^{3}}{\lambda}-\frac{3 \mathrm{t}_{2}^{2}}{\lambda^{2}}+\frac{6 \mathrm{t}_{2}}{\lambda^{3}}-\frac{6}{\lambda^{4}}\right)\right] \\
& -\frac{A \theta^{2}(\alpha) e^{\lambda t_{2}}}{2 \lambda}\left[\begin{array}{l}
\left(\frac{t_{2}^{3}}{\lambda}-\frac{3 t_{2}^{2}}{\lambda^{2}}+\frac{6 t_{2}}{\lambda^{3}}-\frac{6}{\lambda^{4}}\right)-\frac{\theta(\alpha)}{2}\left(\begin{array}{l}
\frac{t_{2}^{5}}{\lambda}-\frac{5 t_{2}^{4}}{\lambda^{2}}+\frac{20 t_{2}^{3}}{\lambda^{3}} \\
-\frac{60 t_{2}^{2}}{\lambda^{4}}+\frac{120 t_{2}}{\lambda^{5}}-\frac{120}{\lambda^{6}}
\end{array}\right) \\
-\frac{2}{\lambda}\left(\frac{t_{2}^{2}}{\lambda}-\frac{2 t_{2}}{\lambda^{2}}+\frac{2}{\lambda^{3}}\right)+\frac{\theta(\alpha)}{\lambda}\left(\frac{t_{2}^{4}}{\lambda}-\frac{4 t_{2}^{3}}{\lambda^{2}}+\frac{12 t_{2}^{3}}{\lambda^{3}}-\frac{24 t_{2}}{\lambda^{4}}+\frac{24}{\lambda^{5}}\right)
\end{array}\right]
\end{aligned}
$$

During period $(0, T)$ total inventory carrying $\left(I_{H}\right)$ can be obtained as

$$
\begin{aligned}
& I_{H}=\int_{0}^{t_{1}} I(t) d t+\int_{0}^{t_{2}} I(t) d t \\
& =K\left(\frac{t_{1}^{2}}{2}-\frac{\theta(\alpha) t_{1}^{4}}{12}-\frac{\theta^{2}(\alpha) t_{1}^{6}}{72}\right)+\frac{A}{\lambda}\left(1+\frac{\theta(\alpha)}{\lambda^{2}}\right)\left(t_{1}-\frac{\theta t_{1}^{3}}{6}\right)-\frac{A e^{\lambda t_{1}}}{\lambda^{2}} \\
& {\left[\left(1+\frac{\theta(\alpha)}{\lambda^{2}}\right)-\theta(\alpha)\left(\frac{t_{1}}{\lambda}-\frac{1}{\lambda^{2}}\right)+\theta^{2}(\alpha)\left(-\frac{10}{\lambda^{5}}+\frac{10 t_{1}}{\lambda^{4}}-\frac{5 t_{1}^{2}}{\lambda^{3}}+\frac{3 t_{1}^{3}}{2 \lambda^{2}}-\frac{t_{1}^{4}}{4 \lambda}\right]\right.}
\end{aligned}
$$




$$
\begin{aligned}
& +\frac{A e^{\lambda t_{2}}}{\lambda} e^{-\theta(\alpha) t_{2}^{2} / 2}\left\{1+\frac{\theta(\alpha) t_{2}^{2}}{2}-\frac{\theta(\alpha) t_{2}}{\lambda}+\frac{\theta(\alpha)}{\lambda^{2}}\right\} t_{2}- \\
& \frac{A e^{\lambda t_{2}}}{\lambda^{2}}\left[\left(1+\frac{\theta(\alpha)}{\lambda^{2}}\right)-\theta(\alpha)\left(\frac{t_{2}}{\lambda}-\frac{1}{\lambda^{2}}\right)+\theta^{2}(\alpha)\left(\begin{array}{l}
-\frac{10}{\lambda^{5}}+\frac{10 t_{2}}{\lambda^{4}}-\frac{5 t_{2}^{2}}{\lambda^{3}} \\
+\frac{3 t_{2}^{3}}{2 \lambda^{2}}-\frac{t_{2}^{4}}{4 \lambda}
\end{array}\right)\right]
\end{aligned}
$$

Total amount of shortage units $\left(\mathrm{I}_{\mathrm{s}}\right)$ during the period $(0, T)$ is given by

$$
I_{S}=-\int_{0}^{t_{3}} I(t) d t+\int_{0}^{t_{4}} I(t) d t=\left[\frac{A}{\lambda}\left\{\left(\frac{e^{\lambda t_{3}}}{\lambda}-t_{3}\right)-\frac{1}{\lambda}\right\}-\frac{K t_{4}^{2}}{2}\right]
$$

Total production $\mathrm{K}_{\mathrm{T}}=\int_{0}^{t_{1}} K d t+\int_{0}^{t_{4}} K d t$

$$
\begin{aligned}
& =\mathrm{K}\left(t_{t}+t_{4}\right) \\
\text { Production cost } & =\mathrm{K} \eta(\mathrm{K})\left(t_{t}+t_{4}\right) \\
& =\left(R K+G+H K^{2}\right)\left(t_{t}+t_{4}\right)
\end{aligned}
$$

Hence the total average cost of the inventory system is

$\mathrm{C}_{\mathrm{T}}=$ Set up cost + Production cost + deterioration cost + inventory carrying cost + shortage cost

$$
=\frac{1}{T}\left[C+\eta(\mathrm{K}) \mathrm{K}_{T}+C_{D} I_{D}+C_{h} I_{H}+C_{S} I_{S}\right]
$$

and putting the value of $\mathrm{I}_{\mathrm{D}, \mathrm{H}} \mathrm{I}_{\mathrm{H}}$ and $\mathrm{I}_{\mathrm{S}}$ we getting the total average cost of the inventory system.

\section{The Approximation Solution Procedure}

$$
\begin{aligned}
& \mathrm{C}_{\mathrm{T}}=\frac{C+\left(R K+G+H K^{2}\right)\left(t_{1}+t_{4}\right)}{T}+\frac{C_{D}}{T}\left[\left\{K \theta(\alpha)\left(\frac{t_{1}^{3}}{6}-\frac{\theta(\alpha) t_{1}^{5}}{15}-\frac{\theta^{2}(\alpha) t_{1}^{7}}{84}\right)\right\}\right]+ \\
& \frac{A \theta(\alpha)}{\lambda}\left(1+\frac{\theta(\alpha)}{\lambda^{2}}\right)\left(\frac{t_{1}^{2}}{2}-\frac{\theta t_{1}^{4}}{8}\right) \\
& -\frac{C_{D} A \theta(\alpha) e^{\lambda t_{1}}}{\lambda T}\left(1+\frac{\theta(\alpha)}{\lambda^{2}}\right)\left[\frac{t_{1}}{\lambda}-\frac{1}{\lambda^{2}}-\frac{\theta(\alpha)}{2}\left(\frac{t_{1}^{3}}{\lambda}-\frac{3 t_{1}^{2}}{\lambda^{2}}+\frac{6 t_{1}}{\lambda^{3}}-\frac{6}{\lambda^{4}}\right)\right] \\
& -\frac{C_{D} A \theta^{2}(\alpha) e^{\lambda t_{1}}}{2 \lambda T}\left[\begin{array}{l}
t_{1}\left(\frac{t_{1}^{3}}{\lambda}-\frac{t_{1}{ }^{2}}{\lambda^{2}}+\frac{6 t_{1}}{\lambda^{3}}-\frac{6}{\lambda^{4}}\right)-\frac{\theta(\alpha)}{2}\left(\begin{array}{l}
\frac{t_{1}^{5}}{\lambda}-\frac{5 t_{1}^{4}}{\lambda^{2}}+\frac{20 t_{1}{ }^{3}}{\lambda^{3}} \\
-\frac{60 t_{1}^{2}}{\lambda^{4}}+\frac{120 t_{1}}{\lambda^{5}}-\frac{120}{\lambda^{6}}
\end{array}\right) \\
-\frac{2}{\lambda}\left(\frac{t_{1}^{2}}{\lambda}-\frac{2 t_{1}}{\lambda^{2}}+\frac{2}{\lambda^{3}}\right)+\frac{\theta(\alpha)}{\lambda}\left(\begin{array}{l}
\frac{t_{1}^{4}}{\lambda}-\frac{4 t_{1}{ }^{3}}{\lambda^{2}}+ \\
\frac{12 t_{1}^{3}}{\lambda^{3}}-\frac{24 t_{t_{1}}}{\lambda^{4}}+\frac{24}{\lambda^{5}}
\end{array}\right)
\end{array}\right] \\
& +\frac{C_{D}}{T}\left[\frac{\theta(\alpha) A e^{\lambda t_{2}}}{\lambda} e^{-\theta(\alpha) t_{2}^{2} / 2}\left(1+\frac{\theta(\alpha) t_{2}^{2}}{2}-\frac{\theta(\alpha) t_{2}}{\lambda}-\frac{\theta(\alpha)}{\lambda}\right) \frac{t_{2}^{2}}{2}\right] \\
& -\frac{C_{D} A \theta(\alpha) e^{\lambda t_{2}}}{\lambda T}\left(1+\frac{\theta(\alpha)}{\lambda^{2}}\right)\left[\frac{t_{2}}{\lambda}-\frac{1}{\lambda^{2}}-\frac{\theta(\alpha)}{2}\left(\frac{\mathrm{t}_{2}^{3}}{\lambda}-\frac{3 \mathrm{t}_{2}^{2}}{\lambda^{2}}+\frac{6 \mathrm{t}_{2}}{\lambda^{3}}-\frac{6}{\lambda^{4}}\right)\right]
\end{aligned}
$$




$$
\begin{aligned}
& -\frac{C_{D} A \theta^{2}(\alpha) e^{\lambda t_{2}}}{2 \lambda T}\left[\begin{array}{l}
\left(\frac{t_{2}^{3}}{\lambda}-\frac{3 t_{2}^{2}}{\lambda^{2}}+\frac{6 t_{2}}{\lambda^{3}}-\frac{6}{\lambda^{4}}\right)-\frac{\theta(\alpha)}{2}\left(\begin{array}{l}
\frac{t_{2}^{5}}{\lambda}-\frac{5 t_{2}^{4}}{\lambda^{2}}+\frac{20 t_{2}^{3}}{\lambda^{3}} \\
-\frac{60 t_{2}^{2}}{\lambda^{4}}+\frac{120 t_{2}}{\lambda^{5}}-\frac{120}{\lambda^{6}}
\end{array}\right) \\
-\frac{2}{\lambda}\left(\frac{t_{2}^{2}}{\lambda}-\frac{2 t_{2}}{\lambda^{2}}+\frac{2}{\lambda^{3}}\right)+\frac{\theta(\alpha)}{\lambda}\left(\frac{t_{2}^{4}}{\lambda}-\frac{4 t_{2}^{3}}{\lambda^{2}}+\frac{12 t_{2}^{3}}{\lambda^{3}}-\frac{24 t_{2}}{\lambda^{4}}+\frac{24}{\lambda^{5}}\right)
\end{array}\right] \\
& \frac{C_{h}}{T} K\left(\frac{t_{1}^{2}}{2}-\frac{\theta(\alpha) t_{1}^{4}}{12}-\frac{\theta^{2}(\alpha) t_{1}^{6}}{72}\right)+\frac{C_{h}}{T} \frac{A}{\lambda}\left(1+\frac{\theta(\alpha)}{\lambda^{2}}\right)\left(t_{1}-\frac{\theta t_{1}^{3}}{6}\right) \\
& -\frac{A e^{\lambda t_{1}}}{\lambda^{2}} \frac{C_{h}}{T}\left[\begin{array}{l}
\left(1+\frac{\theta(\alpha)}{\lambda^{2}}\right)-\theta(\alpha)\left(\frac{t_{1}}{\lambda}-\frac{1}{\lambda^{2}}\right)+ \\
\theta^{2}(\alpha)\left(\begin{array}{l}
-\frac{10}{\lambda^{5}}+\frac{10 t_{1}}{\lambda^{4}} \\
-\frac{5 t_{1}^{2}}{\lambda^{3}}+\frac{3 t_{1}^{3}}{2 \lambda^{2}}-\frac{t_{1}^{4}}{4 \lambda}
\end{array}\right)
\end{array}\right] \\
& +\frac{C_{h}}{T} \frac{A e^{\lambda t_{2}}}{\lambda} e^{-\theta(\alpha) t_{2}^{2} / 2}\left\{1+\frac{\theta(\alpha) t_{2}^{2}}{2}-\frac{\theta(\alpha) t_{2}}{\lambda}+\frac{\theta(\alpha)}{\lambda^{2}}\right\} t_{2}- \\
& \frac{C_{h}}{T} \frac{A e^{\lambda t_{2}}}{\lambda^{2}}\left[\begin{array}{l}
\left(1+\frac{\theta(\alpha)}{\lambda^{2}}\right)-\theta(\alpha)\left(\frac{t_{2}}{\lambda}-\frac{1}{\lambda^{2}}\right)+ \\
\theta^{2}(\alpha)\left(-\frac{10}{\lambda^{5}}+\frac{10 t_{2}}{\lambda^{4}}-\frac{5 t_{2}^{2}}{\lambda^{3}}+\frac{3 t_{2}^{3}}{2 \lambda^{2}}-\frac{t_{2}^{4}}{4 \lambda}\right)
\end{array}\right]+\frac{C_{S}}{T}\left[\frac{A}{\lambda}\left\{\left(\frac{e^{\lambda t_{3}}}{\lambda}-t_{3}\right)-\frac{1}{\lambda}\right\}-\frac{K t_{4}^{2}}{2}\right]
\end{aligned}
$$

In many cases $\lambda$ and $\theta_{0}(\alpha)$ are extremely small hence to use Maclaurin series for approximation

$$
e^{\lambda t}=1+\lambda t+\frac{\lambda^{2} t^{2}}{2}
$$

By using equation (19) the total average cost of system

$$
\begin{aligned}
\mathrm{C}_{\mathrm{T}}=\frac{C+}{\left(R K+G+H K^{2}\right)\left(t_{1}+t_{4}\right)}+\frac{C_{D} K \theta(\alpha) t_{1}^{3}}{6 T}+\frac{A \theta(\alpha) t_{1}^{2}}{2 \lambda} & \\
- & \frac{C_{D} A \theta(\alpha)\left(1+\lambda t_{1}+\frac{\lambda^{2} t_{1}^{2}}{2}\right)}{\lambda T}\left(\frac{t_{1}}{\lambda}-\frac{1}{\lambda^{2}}\right)+\frac{C_{D} \theta(\alpha) A\left(1+\lambda t_{2}+\frac{\lambda^{2} t_{2}^{2}}{2}\right) t_{2}^{2}}{2 \lambda T} \\
- & \frac{C_{D} A \theta(\alpha)\left(1+\lambda t_{2}+\frac{\lambda^{2} t_{2}^{2}}{2}\right)}{\lambda T}\left[\frac{t_{2}}{\lambda}-\frac{1}{\lambda^{2}}\right] \frac{\frac{C_{h}}{T} K\left(\frac{t_{1}^{2}}{2}-\frac{\theta(\alpha) t_{1}^{4}}{12}\right)+\frac{C_{h}}{T} \frac{A t_{1}}{\lambda}\left(1+\frac{\theta(\alpha)}{\lambda^{2}}\right)-}{A\left(1+\lambda t_{1}+\frac{\lambda^{2} \mathrm{t}_{1}^{2}}{2}\right)} \\
& +\frac{C_{h}}{T} \frac{\mathrm{A}\left(1+\lambda t_{2}+\frac{\lambda^{2} t_{2}^{2}}{2}\right) \theta(\alpha)}{\lambda}\left\{\left(1+\frac{\theta(\alpha)}{\lambda^{2}}\right)-\theta(\alpha)\left(\frac{t_{2}}{\lambda}-\frac{1}{\lambda^{2}}\right)\right] \\
& \frac{C_{h}}{T} \frac{A\left(1+\lambda t_{2}+\frac{\lambda^{2} t_{2}^{2}}{2}\right)}{\lambda^{2}}\left[\left(1+\frac{\theta(\alpha)}{\lambda^{2}}\right)-\theta(\alpha)\left(\frac{t_{2}}{\lambda}-\frac{1}{\lambda^{2}}\right)\right]+\frac{C_{S}}{T}\left[\mathrm{~A}\left(\frac{1}{\lambda^{2}}-\frac{t_{3}^{2}}{2}\right)-\frac{1}{\lambda}-\frac{K t_{4}^{2}}{2}\right]
\end{aligned}
$$

According to equation (20) contain five variables $\mathrm{K}, \mathrm{t}_{1}, \mathrm{t}_{2}, \mathrm{t}_{3}$ and $\mathrm{t}_{4}$ and these are dependent variable and related by equation (11) and (12). Also we have $C_{T}>0$, hence the optimum value of $t_{1}, t_{4}$ and $K$ which minimize total average cost are the solutions of the equations 


$$
\frac{\partial C_{T}}{\partial t_{1}}=0, \frac{\partial C_{T}}{\partial t_{4}}=0 \text { and } \frac{\partial C_{T}}{\partial K}=0
$$

Provided that satisfy the conditions

$$
\begin{gathered}
\frac{\partial^{2} C_{T}}{\partial t_{1}^{2}}>0, \frac{\partial^{2} C_{T}}{\partial t_{4}^{2}}>0 \text { and } \frac{\partial^{2} C_{T}}{\partial t_{1}^{2}} \cdot \frac{\partial^{2} C_{T}}{\partial t_{4}^{2}}-\frac{\partial^{2} C_{T}}{\partial t_{1} \partial t_{4}}>0, \\
\frac{\partial^{2} C_{T}}{\partial K^{2}}>0, \frac{\partial^{2} C_{T}}{\partial t_{4}^{2}}>0 \text { and } \frac{\partial^{2} C_{T}}{\partial K^{2}} \cdot \frac{\partial^{2} C_{T}}{\partial t_{4}^{2}}-\frac{\partial^{2} C_{T}}{\partial K \partial t_{4}}>0, \\
\frac{\partial^{2} C_{T}}{\partial t_{1}^{2}}>0, \frac{\partial^{2} C_{T}}{\partial K^{2}}>0 \text { and } \frac{\partial^{2} C_{T}}{\partial t_{1}^{2}} \cdot \frac{\partial^{2} C_{T}}{\partial K^{2}}-\frac{\partial^{2} C_{T}}{\partial t_{1} \partial K}>0 .
\end{gathered}
$$

Now, differentiating (20) with respect to $\mathrm{t}_{1}, \mathrm{t}_{4}$ and $\mathrm{K}$ we get

$$
\begin{aligned}
& \frac{\partial C_{T}}{\partial t_{1}}=\frac{\left(R K+G+H K^{2}\right)}{T}+\frac{C_{D} K \theta(\alpha) t_{1}^{2}}{2 T}+\frac{A \theta(\alpha) t_{1}}{\lambda} \\
& \quad-\frac{C_{D} A \theta(\alpha)\left(1+\lambda t_{1}\right)}{T}\left(\frac{t_{1}}{\lambda}-\frac{1}{\lambda^{2}}\right)-\frac{C_{D} A \theta(\alpha)\left(1+\lambda t_{1}+\frac{\lambda^{2} t_{1}^{2}}{2}\right)}{\lambda^{2} T} \\
& +\frac{C_{D} \theta(\alpha) A\left(2 f\left(t_{1}\right)+3 \lambda f\left(t_{1}\right)^{2}+2 \lambda^{2} f\left(t_{1}\right)^{3}\right) f^{\prime}\left(t_{1}\right)}{2 \lambda T} \\
& -\frac{C_{D} A \theta(\alpha)\left(1+\lambda f\left(t_{1}\right)\right) f^{\prime}\left(t_{1}\right)}{T}\left(\frac{f\left(t_{1}\right)}{\lambda}-\frac{1}{\lambda^{2}}\right)-\frac{C_{D} A \theta(\alpha)\left(1+\lambda f\left(t_{1}\right)+\frac{\lambda^{2} f\left(t_{1}\right)^{2}}{2}\right) f^{\prime}\left(t_{1}\right)}{\lambda^{2} T} \\
& \frac{C_{h}}{T} K\left(t_{1}-\frac{\theta(\alpha) t_{1}^{3}}{3}\right)+\frac{C_{h}}{T} \frac{A}{\lambda}\left(1+\frac{\theta(\alpha)}{\lambda^{2}}\right)+\frac{\theta(\alpha) A\left(1+\lambda t_{1}+\frac{\lambda^{2} \mathrm{t}_{1}^{2}}{2}\right)}{\lambda^{3}} \frac{C_{h}}{T} \\
& -\frac{C_{K} A\left(1+\lambda t_{1}\right)}{T \lambda}\left[\left(1+\frac{\theta(\alpha)}{\lambda^{2}}\right)-\theta(\alpha)\left(\frac{t_{1}}{\lambda}-\frac{1}{\lambda^{2}}\right)\right] \\
& +\frac{A C_{h}}{T} \frac{\left(1+\lambda f\left(t_{1}\right)+\frac{\lambda^{2} f\left(t_{1}\right)^{2}}{2}\right) \theta(\alpha) f^{\prime}\left(t_{1}\right)}{\lambda}\left\{-\frac{2 f\left(t_{1}\right)}{\lambda}+\frac{1}{\left.\lambda^{2}\right\}}\right\}+\frac{\theta(\alpha) A\left(1+\lambda f\left(t_{1}\right)+\frac{\lambda^{2} f\left(t_{1}\right)^{2}}{2}\right) f^{\prime}\left(t_{1}\right)}{\lambda^{3}} \frac{C_{h}}{T} \\
& \frac{A C_{h}}{T}\left(1+\lambda f\left(t_{1}\right)\right) \theta(\alpha)\left\{-\frac{f\left(t_{1}\right)^{2}}{\lambda}+\frac{f\left(t_{1}\right)}{\lambda^{2}}\right\} f^{\prime}\left(t_{1}\right) \\
& -\frac{C_{K} A\left(1+\lambda f\left(t_{1}\right)\right) f^{\prime}\left(t_{1}\right)}{T \lambda}\left[\left(1+\frac{\theta(\alpha)}{\lambda^{2}}\right)-\theta(\alpha)\left(\frac{f\left(t_{1}\right)}{\lambda}-\frac{1}{\lambda^{2}}\right)\right]=0 .
\end{aligned}
$$

$$
\frac{\partial C_{T}}{\partial t_{4}}=\frac{\left(R K+G+H K^{2}\right)}{T}+\frac{C_{S}}{T}\left[-\operatorname{Ag}\left(\mathrm{t}_{4}\right) \mathrm{g}^{\prime}\left(\mathrm{t}_{4}\right)-K t_{4}\right]=0 .
$$

and

$$
\begin{aligned}
& \frac{C_{D}}{T}\left[\left\{\theta(\alpha)\left(\frac{t_{1}^{3}}{6}-\frac{\theta(\alpha) t_{1}^{5}}{15}-\frac{\theta^{2}(\alpha) t_{1}^{7}}{84}\right)\right\}\right]+\frac{C_{h}}{T}\left(\frac{t_{1}^{2}}{2}-\frac{\theta(\alpha) t_{1}^{4}}{12}-\frac{\theta^{2}(\alpha) t_{1}^{6}}{72}\right) \\
- & \frac{C_{S} K t_{4}^{2}}{2 T}+\frac{(R+2 H K)\left(\mathrm{t}_{1}+\mathrm{t}_{4}\right)}{T}=0 .
\end{aligned}
$$

If $\theta(\alpha)<<1$ then

$$
K=-\frac{\theta(\alpha)\left[\mathrm{R}+C_{D} t_{1}^{3}+3 C_{h} f^{2}\left(t_{1}\right)-3 C_{S} t_{4}{ }^{2}\right]}{12 H\left(\mathrm{t}_{1}+\mathrm{t}_{4}\right)}
$$

And optimal production rate,

$$
K^{*}=-\frac{\theta(\alpha)\left[\mathrm{R}+C_{D} t_{1}^{* 3}+3 C_{h} f^{2}\left(t_{1}^{*}\right)-3 C_{S} t_{4}^{* 2}\right]}{12 H\left(\mathrm{t}_{1}^{*}+\mathrm{t}_{4}{ }^{*}\right)}
$$

Here we obtain three simultaneous non-linear equation in $t_{1}, t_{4}$ and $K$. Optimal value of $t_{1}, t_{4}$ and $K$ can be find out by using some suitable computational numerical method and the optimum value of $t_{2}, t_{3}, I_{m}, I_{b}$ and minimum total average cost ' $\mathrm{C}_{\mathrm{T}}$ ' can be obtained from the above equation. 


\section{Special Cases:}

Case $\mathbf{i}:$ If $\theta_{0}(\alpha)=0$ then the discussed model reduces to production inventory model with out deterioration

Case ii : If $\lambda=0$, the model reduce to constant demand.

\section{Conclusion :}

In the proposed model a production inventory model is formulated for random deteriorating item with a increasing market demand rate with time and production rate is variable. Result in this study can provide a valuable reference for decision markers in planning the production and controlling the inventory. The model proposed here in is resolved by using maclaurin series and cost minimization technique is used to get the approximate expression for total average cost and other parameters \& some special cases of model are also discussed.A future study will incorporate more realistic assumption in the proposed model.

\section{References}

[1]. Alder, . G. L and R. Nanda, (1974) The effects of learning on optimal lot size determination- single product case, AIIE Trans. 6, 14-20.

[2]. Axster, S. and S. E. Elmaghraby, (1981) A note on EMQ under learning and forgetting,AIIE Trans. 13,86-90.

[3]. Baker, R. C. and Urban, T. L., (1988) A deterministic inventory system with an inventory-level-dependent demand rate, Opl.Res. Soc.39, $823-831$.

[4]. Balkhi, Z. T. and Benkherouf, L. (1996 b) On the optimal replenishment schedule for an inventoy system with deteriorating items and time varying demand and production rates. Computers \& Industrial Engineering, 30; 823-829.

[5]. Bhunia,A.K.and Maiti,M. (1997) Deterministic inventory models for variable production. Jour of Opi. Res. Soc., $48: 221-224$.

[6]. Bose, S., Goswami, A and Chaudhuri, K.S. (1995) An EOQ model for deteriorating items with linear time dependent demand rate and shortages unde inflation and time discounting.Jour Of Opl, Res. Soc., 46:507-728.

[7]. Chang, H. J. and Dye, C. Y. (1999)An EOQ model for deteriorating items with time varying demand and partial backlogging.Jour, of Opl. Res. Soc., 50:1176-1182.

[8]. Cheng, T. C. (1991) An economic order quantity model with demand dependent unit production cost and imperfect production processes, IIE Trans.23, 23-28.

[9]. Covert, R. P. and Philip, G. C. (1973) An EOQ model for items with weilbull distribution deterioration. A1TE Trans, 5 (4) : $323-329$.

[10]. Datta, T. K. and Pal, A. K., (1990) note on an inventory model with inventorylevel-dependent demand rate, Opl.Res. Soc. 41 ,971-975.

[11]. Gallego, G. (1993) Reduced production rates in the economic lot scheduling problem, Int. J. Prod. Res. 31, $1035-1046$.

[12]. Ghare, P. M. and Scharder, G. F. (1963) A model for exponentially decaying inventories.Jour. Ind. Engg., 14 : 238-243.

[13]. Giri, B. C. , Pal, S. ,Goswami, A. and Chaudhuri, K. S., (1995) An inventory model for deteriorating items with stock-dependent demand rate, Euro.J. Oper. Res.95, 604-610.

[14]. Giri, B. C. and Chaudhuri, K. S., Deterministic models of perishable inventory with stock-dependent demand rate and nonlinear holding cost, Euro. J. Oper. Res.105 (1998) 467-474.

[15]. Goh, M. , (1994) EOQ models with general demand and holding cost functions,Euro. J. Oper. Res.73,50-54

[16]. Goswami, A and Chaudhuri, K. S. (1991) EOQ model for an inventory with a linear trend in demand and finite rate of replenishment considering shortages.int. Jour. Syst ScL, 22:181-187.

[17]. Hax, A. C. and D. Candea, ,(1984) Production and Inventory Management,Prentice-Hall, Englewood Cliffs, NJ

[18]. Khouja, M. and Mehrez, A. , (1994) An economic production lot size model with imperfect quality and variable production rate, Opl. Res. Soc.45, 1405-1417.

[19]. Khouja, M., (1995) The economic production lot size model under volume flexibility, Computers Ops. Res. 22 ,515-523.

[20]. Mondal , B. N. and Phaujder, S. (1989) An inventory model for deteriorating items and stock-dependent demand rate, Opl. Res. Soc. 40, 483-488.

[21]. Moon, I. , Gallego, G. and Simchi-levi, D., (1991) Controllable production rates in a family production context, Prod.Res.29, $2459-2470$.

[22]. Muth, E. J. and K. Spearmann, (1983) Learning effects in economic lotsize, Mgmt Sci. 29, 264-269.

[23]. Proteus, E. L., (1986) Optimal lot sizing, process quality improvement, and setup cost reduction, Ops. Res. 34, 137-144.

[24]. Yadav, R.K., and Yadav, P.(2013) Volume flexibility in production model with cubic demand rate weibull deterioration with partial backlogging, IOSR Journal of mathematics Volume 6,29-34.

[25]. Rosenblatt, M. J. and Lee, H. L., (1986) Economic production cycles with imperfect production processes, IIE Trans. 17 ,48-54.

[26]. Roy, J. and Chaudhuri, K. S., (1997) An EOQ model with stock-dependent demand, shortages, inflation and time discounting, Int. J. Prod. Eco. $53,171-180$.

[27]. Roy, J. , Goswami, A. and. Chaudhuri, K. S., (1998) On an inventory model with two levels of storage and stock-dependent demand rate, Int. J.Systems. Sci.29,249-254.

[28]. Sana, S. and Chaudhuri, K. S., (2003)On a volume flexible stock-dependent Inventory model, Advanced Modeling and Optimization, Volume 5, Number 3.

[29]. Sethi, A. K. and Sethi, P. S. Flexible in Manufacturing: A Survey, Int.J. Flexible Manufact.Systems.2 (1990) $289-328$.

[30]. Schweitzer, P. J. and Seidman, (1991) A. Optimizing processing rates for flexible manufacturing systems, Mgmt. Sci. $37454-466$.

[31]. Silver, E. A., (1990) Deliberately slowing down output in a family production context, Int. J. Prod. Res. 28 17-27.

[32]. Silver, E. A. and Peterson, R., .(1985) Decision Systems for Inventory Managementand Production Planning, Wiley, New York, 2nd edn

[33]. Silver, E. A., (1979) A simple inventory replenishment decision rule for a linear trend in demand, Opl. Res. Soc.30 $71-75$.

[34]. Sule, D. R. (1981) A note on production time variation in determining EMQ under influence of learning and forgetting, AIIE Trans. 13,9195.

[35]. Sule, D. R., (1978) The effect of a alternate periods learning and forgetting on economic manufacturing quantity, AIIE Trans. 10 ,338-343.

[36]. Urban, T. L., (1992) An inventory model with an inventory-level-dependentdemand rate and relaxed terminal condition, Opl. Res. Soc. 43 ,721-724.

[37]. Zoutendijk, G., (1966) Nonlinear Programming: A Numerical Survey, SIAM J. Control. 4 ,194-210. 